\title{
View of Self Scale: Measuring Negative Self-Referential Thoughts in Depression
}

\author{
Jennifer K Lehmann, Ph.D*, James COverholser, Ph.D, Alison Athey, Ph.D, Silvia Hernandez, \\ M.A, Eleanor Beale, M.A and Josephine Ridley, Ph.D
}

Department of Psychological Sciences, Case Western Reserve University, Ohio, USA

\begin{abstract}
Negative beliefs regarding the self are a key feature of depression, over and above beliefs about the future or beliefs about the world. A clinically relevant measure of self-view could help further understanding of the relationship between depression and an individual's view of self. Assessing negative beliefs about the self could also help clinicians identify targets for treatment and monitor progress during psychotherapy for depression. The present study provided an initial investigation of the reliability and validity of the View of Self Scale (VOSS), a new theoretically-derived measure designed to assess negative beliefs about the self. Theoretically-derived subscales focus on cognitions of self-worth, competence, and social desirability. The VOSS was administered to two samples: A clinical sample of depressed veterans recruited from a psychiatric outpatient clinic and partial hospitalization program (PHP) at a Veterans Affairs Medical Center, and a community sample of adults collected from food pantries in a large Midwestern city. The VOSS demonstrated strong psychometric properties in the present samples. Participants in the clinical sample reported significantly more negative self-views than the community adult sample. Self-view scores exhibited large correlations with depression severity in both depressed patients and community adults. VOSS scores were also significantly related to age in the clinical sample, but not in the community sample. The findings from this study reinforce that psychotherapeutic interventions focused on addressing self-view, such as cognitive behavioral therapy, may help to prevent or reduce the symptoms of depression.
\end{abstract}

\section{Introduction}

The way in which individuals view, conceptualize, and evaluate themselves has important implications for both physical and emotional functioning. Feelings of self-worth or self-regard are linked to indicators of psychological adjustment such as happiness [1]. Individuals with positive self-views are more likely to respond to negative events with greater perceived control, greater optimism about their coping abilities, and less overall distress [2]. In response to acute stress, individuals reporting higher self-evaluations exhibit reduced stress reactivity via lower heart rates and reduced inflammatory responses [3]. Additionally, in a study of 502 adults who were over the age of 60 years old and diagnosed with cardiovascular disease, female participants with positive self-views demonstrated better physical and psychosocial functioning after one year [4].

View of self has been implicated in the definition, development, course, and treatment of psychological disorders. Both negative self-views and positive or inflated self-views can be associated with different forms of psychological distress. Negative self-views are common in individuals with depressive disorders, anxiety disorders, schizophrenia, and other psychiatric conditions $[5,6]$. Individuals with narcissistic personality disorder may exhibit an inflated explicit self-view while maintaining a negative implicit view of themselves [7].
Despite the frequency of negative self-views in multiple psychiatric conditions, depression may be uniquely characterized by low self-worth more than other diagnoses [8].

Negative evaluations of the self also reflect a causal risk factor for depression $[9,10]$. Theories consistent with the vulnerability model of depression conceptualize negative evaluations of the self as reflecting a relatively stable cognitive factor, predisposing a person to experience depression. Other variables, however, are able to mediate or moderate the relationship between risk factors for depression and the occurrence of a depressive episode [11]. Individuals with temporally unstable or labile self-evaluations may be especially at risk for developing depressive symptoms [12].

Negative self-perceptions may be related to suicidal ideation and attempts. In a sample of 338 psychiatric out-

*Corresponding author: Jennifer K Lehmann, Ph.D., Department of Psychological Sciences, Case Western Reserve University, Ohio, USA

Accepted: May 04, 2021

Published online: May 06, 2021

Citation: Lehmann JK, Overholser JC, Athey A, et al. (2021) View of Self Scale: Measuring Negative Self-Referential Thoughts in Depression. Ann Cogn Sci 5(1):185-195 
Citation: Lehmann JK, Overholser JC, Athey A, et al. (2021) View of Self Scale: Measuring Negative Self-Referential Thoughts in Depression. Ann Cogn Sci 5(1):185-195

patients, both negative beliefs about oneself and negative beliefs about how other people regard oneself were associated with suicidal ideation after controlling for depression and hopelessness [13]. Suicide attempters have shown lower levels of self-esteem than non-attempters, even when there were no significant group differences in overall depressive symptoms [14]. In a sample of 254 adolescent psychiatric inpatients and 288 high school students, low self-esteem was related to higher levels of depression, hopelessness, suicidal ideation, and an increased likelihood of having previously attempted suicide [15].

The present study provided an initial investigation of the development of a measure focused specifically on the view of self, defined as an individual's extended review and evaluation of their own personality traits, moral standards, physical appearance, social skills, and inherent merit as a person. The View of Self Scale (VOSS) attempts to address the need for a theoretically grounded measure that addresses the view of self facet of Beck's cognitive theory in a way that is both independent of the other facets of the theory and also more comprehensive than other present measures of view of self. Other established measures that confront self-esteem (e.g., Rosenberg Self-Esteem Scale) focus on broad views of self that are not amenable to therapeutic change. Such items appear expansive and vague to most clients (e.g., "I am able to do things as well as most people"). Other measures (e.g., Cognitive Triad Inventory) stray more broadly and attempt to examine all three components of the cognitive triad (view of self, view of the world, view of the future). Some of the Cognitive Triad Inventory items are framed in a manner that is unrealistic in most clinical settings (e.g., "I have many talents and skills").

Further, in many clinical situations, depressed clients struggle with a negative view of self while not reporting pessimistic views of the future. Thus, this study explored the development of a brief, focused, and psychometrically sound measure of a client's view of self can have value in both research settings and clinical services.

\section{Method}

\section{Participants}

Adult psychiatric patients: The sample included adult psychiatric outpatient military veterans $(n=47)$ recruited from a large, urban, Midwest VA Medical Center. Participants were recruited from a partial hospitalization program (PHP) for psychiatric patients and an outpatient mental health clinic. Psychiatric participants were included in the study if the diagnostic interview (SCID-I v2.0) [16] indicated that they met DSM- IV criteria [17] for a current depressive disorder including MDD-Recurrent Episode, MDD-Single Episode, Dysthymia, Adjustment Disorder with Depressed Mood, or Depression-Not Otherwise Specified (NOS). Participants were included if they had a depressive disorder and a commonly co-occurring disorder (e.g., posttraumatic stress disorder [PTSD], substance dependence, panic disorder, or generalized anxiety disorder [GAD]) due to the high likelihood of comorbid psychiatric disorders in both the present sample and the general population [18]. Participants meeting criteria for psychotic disorders, bipolar disorders, or dementia were excluded from the study.

A total of 55 patients provided informed consent to participate in the study. Eight participants from the veteran sample were excluded from analyses for meeting study exclusion criteria following completion of study procedures $(n=6,11 \%)$, unreliable reporting ( $n=1,2 \%)$, or for missing more than three measure items $(n=1,2 \%)$.

The final veteran sample included 47 participants. The veteran sample was primarily Male $(n=38,80.8 \%)$ and white ( $n=23,48.9 \%$ ). Participants ranged in age from 26 to 74 years old $(M=53.53, S D=12.89)$. Participants typically met criteria for Major Depression-Recurrent ( $n=28,60 \%$ ) or Major Depression-Single Episode ( $n=16,34 \%)$. Three patients met criteria for a depressive disorder in partial remission at the time of the study procedures. Participants presented with a number of comorbid diagnoses including, PTSD ( $n=26,55 \%)$, Alcohol Dependence ( $n=10,21 \%$ ), Substance Dependence ( $n=7,15 \%)$, Generalized Anxiety Disorder $(n=4,9 \%)$ and Dysthymia $(n=4,9 \%)$.

Community adults: The study also included a community sample of adults presenting to two participating food pantries from a large, urban Midwest city $(n=39)$. Participants were recruited while entering, exiting, or waiting in line for services. Participants recruited from food pantry sites did not complete a diagnostic interview as part of study procedures.

A total of 40 community adult participants consented to the study. One participant $(n=1,3 \%)$ was excluded from analyses for observed lack of effort and an inconsistent response pattern to study questionnaire items. The overall community adult sample included 39 participants (Male: $n=15$, Caucasian: $n=17$ ) ranging in age from 26 to 82 years old $(M=$ $56.15, S D=13.57)$. Of the 40 community adult participants in the study, 21 (53\%) participants were collected from Site A and 19 (47\%) were collected from Site B. The community adult sample from Site $A$ included 21 participants (Male: $n=$ 11 , Caucasian: $n=10$ ) ranging in age from 26 to 82 years old $(M=55.85, S D=13.37)$. The community adult sample from Site B included 19 participants (Male: $n=4$, Caucasian: $n=7$ ) ranging in age from 32 to 76 years old $(M=56.47, S D=14.13)$.

\section{Measures}

The structured clinical interview for the DSM-IV (SCID v2.0) [16] is a structured diagnostic interview designed to assess the presence of psychiatric diagnoses in accordance with DSM-IV criteria (American Psychiatric Association) [17]. The SCID has exhibited adequate reliability and validity. The SCID-I has demonstrated high test-retest reliability between 0.84 and 1.00 within an interval of $78.5 \pm 53$ days for Axis I disorders [17]. Inter-rater reliability for SCID diagnoses ranges from moderate $(k=0.60)$ to excellent $(k=0.81)$ for Axis I disorders [19]. Kappa values for diagnosis of MDD range from 0.66 to 0.70 [20]. The current study utilized a modified version of the SCID-I to focus on diagnoses of depressive disorders while screening for other Axis-I disorders. Graduate students trained in administering the SCID-I completed diagnostic interviews. Training included viewing a SCID-IV train- 
Citation: Lehmann JK, Overholser JC, Athey A, et al. (2021) View of Self Scale: Measuring Negative Self-Referential Thoughts in Depression. Ann Cogn Sci 5(1):185-195

ing video, being walked through administration by previously trained students, observing three administrations completed by a trained interviewer to learn proper administration, and being observed while administering the SCID-I during the training period.

The View of Self Scale (VOSS) was designed to measure three specific domains of self- image: Self-worth, competence, and social desirability. The VOSS aims to meet scale development criteria established by Keith \& Bracken [21]. The VOSS is developed based on a strong theoretical foundation in the cognitive theory of depression. The measure provides a clear operational definition for the view of self as derived from Beck's [9] conceptualization of self-concept. The VOSS is a self-report measure of stable state-self views and utilizes closed-format items based on a 5-point Likert scale. Uniform, fixed-response categories are provided to the patient rather than allowing for open response options to increase ease of response for the patients and ease of scoring for the clinician or researcher. Items were adapted in part from current measures of general depressogenic cognitions or attitudes. Self-referential items were used from Young's Schema Questionnaire [22], the Cognitive Triad Inventory [23], the Rosenberg Self-Esteem Scale [24], and Beck's Depression Checklist [25]. Some items were revised for length, clarity, and reading level. The first draft of the measure included 33 generated items, such as "I feel like I am flawed and defective" (SelfWorth Subscale), "I feel like I can't do anything right" (Competence Subscale), and "I feel that I'm not lovable" (Social Desirability Subscale) and The 33-item measure was provided to the study team of two clinical psychologists, four clinical psychology graduate students, and a psychiatric nurse practitioner. Study team members assessed the items for completeness of the operational definition of the construct, the relevance and clarity of individual items, and potential untapped dimensions of the construct. Following feedback from the research team, social isolation/social irritability and guilt/ deservingness items were added and two redundant items were removed. Additionally, some items were reverse-scored to reduce the emotional impact of the measure with numerous negative statements and to minimize the danger of acquiescent response bias. The final version of the scale has 33 items. Higher scores indicate lower self-view or higher negative self-related thoughts.

The Cognitive Triad Inventory (CTI) [23] includes 36 items measuring patient beliefs regarding view of self, view of the world, and view of the future. Each domain is measured with ten self-report items, phrased in both the positive and negative direction. The CTI included in the present study was modified to remove six filler items. Individuals rate items on a 7-point Likert scale from 1 ("total agree") to 7 ("totally disagree"). Before summing individual item scores, positively-worded items are reversed-scored so that all items are in the same direction of valence in which lower scores represent positive views and higher scores represent negative views. Higher scores indicate lower self-view or higher negative self-related thoughts.

The subscales of the CTI have demonstrated good to excellent internal reliability in an outpatient mental health setting with alpha coefficients of 0.91 for View of Self, 0.81 for View of World, and 0.93 for View of Future [23]. The total scale has demonstrated excellent internal consistency with an alpha of 0.95 . Total CTI scores have been shown to correlate highly with BDI scores ( $r=0.77)$ [23]. The CTI-Self subscale was included in the present study analyses rather than the total scale. Investigations into the factor structure of the CTI in nonclinical samples have been unable to confirm the 3-factor model suggested by the authors [26]. Additional investigations have identified alternative models including a 6-factor model [26], 5-factor model [27], or one-factor model [28].

The Rosenberg Self-Esteem Scale (RSES) [24] is a ten-item self-report measure designed to assess the presence or lack of self-esteem. Items on the RSES are scored on a four-point Likert-type scale ranging from strongly agree to strongly disagree. On the RSES, five items include positively worded statements and five items include negatively worded statements. Before summing individual item scores, positively-worded items are reversed-scored so that all items are in the same direction of valence. The RSES is scored so that higher scores indicate higher self-esteem and lower scores indicate lower self-esteem. Total scores on the RSES range from 0 to 30 . The Rosenberg Self-Esteem Scale has demonstrated good internal consistency with an alpha coefficient of 0.86 [29]. The RSES has also demonstrated a strong, negative relationship with depressive symptoms and negative thinking [30]. The Rosenberg Self Esteem Scale was intended to be a unitary measure of self-esteem, but it may reflect two underlying constructs. Self-esteem reflected in negative comments, or negative evaluations of self, may have greater relevance to the development of depression whereas self-esteem reflected in positive statements may have greater implications in the recovery from depression [31]. Global measures of self-esteem such as the RSES may not be appropriate for use in predicting the development of depression symptoms after accounting for prior depression symptoms [32].

The Patient Health Questionnaire (PHQ-9) [33] measures current symptoms of depression. The PHQ-9 is a 9-item self-report measure of the frequency and severity of present depressive symptoms based on DSM-IV depression diagnostic criteria. Items are scored on a Likert-type scale ranging from 0 (not at all) to 3 (nearly everyday). The measure also includes an item focused on functional impairment caused by depression symptoms in work, relationships, and household tasks. The functional impairment item is scored by selecting one of four responses (not difficult at all, somewhat difficult, very difficult, or extremely difficult). The total severity score is calculated from the sum of the nine symptom items. Total scores range from 0 to 27.

Severity scores between 5 and 9 indicate minimal symptoms of depression, scores between 10 and 14 suggest mild forms of depression, scores from 15 to 19 indicate a moderately severe major depressive episode, and scores higher than 20 indicate severe depression. Cutoff scores ranging from 8 to 11 show no significant differences in the ability to screen for depression [34]. In a primary care setting, the PHQ9 has demonstrated excellent internal consistency, with a Cronbach's alpha score of 0.89 [33]. Test-retest reliability for 
Citation: Lehmann JK, Overholser JC, Athey A, et al. (2021) View of Self Scale: Measuring Negative Self-Referential Thoughts in Depression. Ann Cogn Sci 5(1):185-195

the PHQ-9 within 48 hours was also excellent with a correlation of 0.84 between scores at both time points. The PHQ-9 has demonstrated strong correlations with other measures of depression including the Hospital Anxiety and Depression Scale (HADS) $(r=0.68)$ [35].

The Modified Life Experiences Survey (MLES) is a self-report measure of both recent and past stressors. The MLES was adapted from the Life Experience Survey (LES) [36] and consists of 21 stressful life events. Events in the survey include interpersonal difficulties, health issues, death of loved ones, occupational problems, and acts of violence. Responses are rated on a scale from 0 to $3(0=$ never, $1=$ long ago, $2=$ recently, 3 = long ago and recently). The original LES demonstrates moderate test-retest reliability ( $\alpha=0.65$ ) [36] over a 5 to 6 -week time period. Reliability coefficients for the negative change score ranged from 0.56 to 0.88 [36]. The original LES negative change scores have shown convergent validity through significant correlations with measures of anxiety ( $r=$ 0.29) and depression ( $r=0.24)$ [36].

\section{Procedure}

VAMC procedure: After completing informed consent procedures, participants were administered the SCID-I to determine diagnostic eligibility. Participants also completed a packet of self-report questionnaires. All participants who completed the SCID-I or the full assessment were given a small remuneration of a $\$ 10$ gift card to either Wal-Mart or Amazon.com.

Community social service agencies: Participants were recruited from two IRB-approved food pantry sites. The research assistant verbally described the study procedures and exclusion criteria to a group of patrons in the waiting room of each food pantry site. In one site, the study description was translated into Spanish by a staff member. At each site, the research assistant noted that study procedures required study participants to be able to read and write in English.

The research assistant described the study and obtained informed consent. Participants completed study measures under the observation of the research assistant. Food pantry participants did not complete a SCID interview but did complete all other measures for the present study. All participants who completed the questionnaires received a small remuneration of a \$10 gift card. Due to the inability of study staff to identify participants after completing study procedures and the nature of the questionnaire items focused on depression and suicide, all participants were supplied with a list of contact information and free or sliding scale clinics near the data collection sites prior to initiating study procedures.

\section{Results}

All statistical analyses were conducted with SPSS 25.0. Missing data were rare and Little's MCAR test showed that data were missing at random [37]. Data from one participant was removed from analyses because three or more values were missing. Eight participants were missing only one $(n=$ $6)$ or two items ( $n=2)$ across all scales. Cases with missing demographic variables were excluded pairwise, meaning they were only excluded from analyses that required the missing data. For subjects missing only one or two items, intermediate response options were inserted in place of missing values $(\mathrm{PHQ}-9=1.5, \mathrm{CTI}=4$, VOSS $=2, \mathrm{MLES}=1.5, \mathrm{RSES}=1.5)$.

Table 1: Demographic variables among clinical sample and community adults.

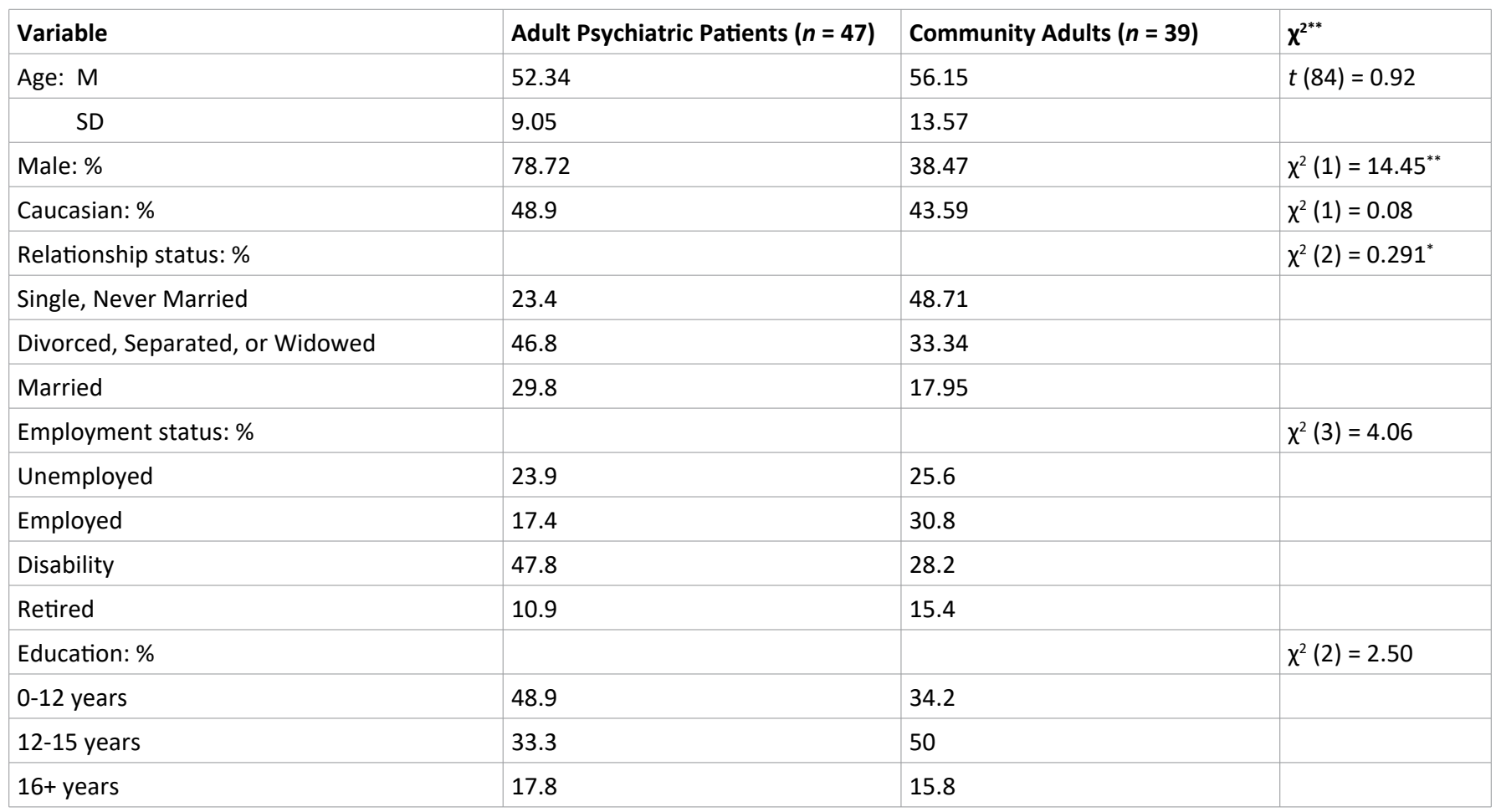

Note: ${ }^{*} p<0.05,{ }^{* *} p<0.0001$

All analyses are $\chi^{2}$ except for age, which is a t-test. 
Citation: Lehmann JK, Overholser JC, Athey A, et al. (2021) View of Self Scale: Measuring Negative Self-Referential Thoughts in Depression. Ann Cogn Sci 5(1):185-195

Depressed patients from a VA medical center $(n=47)$ and community adults utilizing food pantry services $(n=39)$ were compared on a number of demographic variables (see Table 1 ). Participants from both food pantries were combined for comparisons between community adult participants and clinical patients. An independent samples $t$-test showed there was no significant difference between groups in terms of age. A series of chi-square tests of independence were conducted to assess for group differences on race, marital status, employment status, and educational attainment. There were no significant differences between groups on age, race, employment status, or educational attainment. However, the community adult sample consisted of significantly more female participants than the clinical sample $\left(\chi^{2}(1)=14.45, p<0.001\right)$. Additionally, a greater number of individuals in the community adult sample were single and never married than the clinical sample $\left(\chi^{2}(2)=0.291\right.$, $p=0.026)$.

Participants in the clinical outpatient sample and, to a lesser extent, the community adult sample reported a considerable amount of psychopathology. Clinical participants reported moderately severe levels of depression and a high proportion of the sample endorsed a history of one or more prior suicide attempt(s) ( $n=22,47 \%)$. Within the clinical sample, $21 \%$ of participants disclosed experiencing suicidal ideation within days of completing study procedures $(n=10$, $21 \%)$. Additionally, $33 \%$ of the current clinical participants endorsed past psychiatric hospitalization. Within the community sample, nine participants $(23 \%)$ endorsed current pharmacological ( $n=1,3 \%)$, psychological $(n=2,5 \%)$, or both pharmacological and psychological $(n=6,15 \%)$ treatments for depression. Additionally, seven community participants (18\%) endorsed receiving past medication or counseling for depression, but no current treatment. A history of at least one suicide attempt was reported by $21 \%(n=8)$ of the community sample.

The clinical sample of veterans and community sample of adults utilizing food pantry services were also compared on self-report questionnaires (see Table 2). Patients in the clinical sample reported a higher incidence of negative self-related thoughts as evidenced through both the CTI-Self sub- scale $(t(84)=5.39, p<0.001)$ and the VOSS $(t(84)=5.09, p<$ $0.001)$ compared with the community adult sample. Similarly, clinical patients reported lower self-esteem through the RSES $(t(84)=6.99, p<0.001)$ than community adult participants. Clinical patients also reported higher levels of depression ( $t$ $(84)=7.56, p<0.001)$ and life stress $(t(84)=3.01, p<0.01)$ than community adults. Although analyses suggest groups are similar on a number of demographic variables, important differences between groups on all dependent measures preclude the combination of samples for statistical analyses.

\section{VOSS scale development}

All scale reduction and item analysis techniques were conducted within the clinical sample. The 33 items of the VOSS were subjected to exploratory factor analysis (EFA). Prior to performing EFA, the suitability of the data for factor analysis was assessed. The strength of inter-correlations among VOSS items was assessed for evidence of coefficients greater than 0.30 . Initial analyses demonstrated the presence of many coefficients greater than 0.30 , supporting the use of factor analytic techniques with the data. The Kaiser-Meyer-Olkin value was 0.84 , exceeding the recommended value of 0.60 [38] and Bartlett's Test of Sphericity [39] reached statistical significance, supporting the factorability of the correlation matrix. Despite early indicators of factorability of the data, the factor solution produced from the analysis was invalid due to the presence of an ultra-Heywood case. Scale reduction and item analysis were instead completed using classical test theory techniques.

Item-level analyses were conducted for the VOSS. The distribution of responses for each item was examined to assess item performance within the measure. Item response frequencies were examined for a high level of non-response, low variability, floor effects, and ceiling effects (see Table 3). Two items (Items AA and $\mathrm{H}$ ) were removed because they evidenced low variability (fewer than $5 \%$ of responses in any cell of potential item responses). No other issues related to floor effects, ceiling effects, or non-response were identified.

Item-total, item-subscale total, and inter-item correlations were checked for the VOSS. Items with item-total or

Table 2: Dependent measures among depressed day hospital patients and community adults.

\begin{tabular}{|c|c|c|c|c|}
\hline \multirow{2}{*}{$\begin{array}{l}\text { Variable } \\
\text { View of Self Scale }\end{array}$} & \multirow[b]{2}{*}{$\mathrm{M}$} & \multirow{2}{*}{\begin{tabular}{|l|} 
VA Patients \\
46.45
\end{tabular}} & \multicolumn{2}{|c|}{ Community Adults } \\
\hline & & & 22.23 & $t(84)=5.09^{* *}$ \\
\hline & SD & 27.03 & 13.41 & \\
\hline \multirow[t]{2}{*}{ Patient Health Questionnaire } & $\mathrm{M}$ & 16.09 & 5.92 & $t(84)=7.56^{* *}$ \\
\hline & SD & 6.69 & 5.58 & \\
\hline \multirow[t]{2}{*}{ Rosenberg Self-Esteem Scale } & $\mathrm{M}$ & 14.61 & 23.59 & $t(80.75)=7.25^{* *}$ \\
\hline & SD & 6.83 & 4.59 & \\
\hline \multirow[t]{2}{*}{ Cognitive Triad Inventory-Self } & M & 39.06 & 22.82 & $t(84)=5.39^{* *}$ \\
\hline & SD & 14.46 & 9.75 & \\
\hline \multirow[t]{2}{*}{ Modified Life Experiences Survey } & $\mathrm{M}$ & 21.79 & 16.6 & $t(84)=3.01^{*}$ \\
\hline & SD & 7.33 & 8.7 & \\
\hline
\end{tabular}

Note: ${ }^{*} \mathrm{p}<0.05,{ }^{* *} \mathrm{p}<0.0001$ 
Citation: Lehmann JK, Overholser JC, Athey A, et al. (2021) View of Self Scale: Measuring Negative Self-Referential Thoughts in Depression. Ann Cogn Sci 5(1):185-195

Table 3: Distribution of responses for each item of the view of self scale in a sample of depressed patients.

\begin{tabular}{|c|c|c|c|c|c|}
\hline \multirow[t]{2}{*}{ Item } & \multicolumn{5}{|c|}{ Response Options (\%) } \\
\hline & 0 & 1 & 2 & 3 & 4 \\
\hline I think I am a failure in my important relationships. & 23.4 & 19.1 & 6.4 & 27.7 & 23.4 \\
\hline $\begin{array}{l}\text { I think that almost nothing I do at work (or school or household chores) is as } \\
\text { good as what other people can do. }\end{array}$ & 36.2 & 17 & 17 & 14.9 & 14.9 \\
\hline I feel that I deserve to be loved. & 36.2 & 10.6 & 19.1 & 19.1 & 14.9 \\
\hline I feel like something is fundamentally wrong with me. & 12.8 & 14.9 & 10.6 & 29.8 & 31.9 \\
\hline I feel that I am loved by friends, family, and/or my significant other. & 23.4 & 23.4 & 17 & 27.7 & 8.5 \\
\hline I feel that I have let people down in some important ways. & 8.5 & 12.8 & 21.3 & 19.1 & 38.3 \\
\hline I feel like I screw up everything I try. & 23.4 & 17 & 21.3 & 21.3 & 17 \\
\hline I think I am too unlovable to reveal myself to other people. & 38.3 & 4.3 & 23.4 & 19.1 & 14.9 \\
\hline When people show interest in me, I feel I am fooling them. & 34 & 19.1 & 14.9 & 12.8 & 19.1 \\
\hline I think I am not important in any way. & 38.3 & 10.6 & 21.3 & 14.9 & 14.9 \\
\hline I see myself as physically attractive. & 106 & 17 & 17 & 27.7 & 27.7 \\
\hline I feel that I am worthless. & 29.8 & 25.5 & 17 & 10.6 & 17 \\
\hline I think I am no good at all. & 36.2 & 17 & 21.3 & 12.8 & 12.8 \\
\hline I have little respect for myself. & 34 & 14.9 & 23.4 & 14.9 & 12.8 \\
\hline I like myself as a person. & 17 & 25.5 & 25.5 & 21.3 & 10.6 \\
\hline I feel like I am a loser in every way. & 36.2 & 8.5 & 25.5 & 14.9 & 14.9 \\
\hline I think that I drive people away from me. & 21.3 & 17 & 19.1 & 12.8 & 29.8 \\
\hline I believe that I am not worthy of people's attention or affection. & 31.9 & 10.6 & 21.3 & 19.1 & 17 \\
\hline I feel that I'm not lovable. & 38.3 & 10.6 & 14.9 & 17 & 19.1 \\
\hline I feel like I am flawed and defective. & 29.8 & 12.8 & 23.4 & 17 & 17 \\
\hline I think that I do not have much to be proud of. & 23.4 & 23.4 & 25.5 & 8.5 & 19.1 \\
\hline $\begin{array}{l}\text { I believe that no one I desire would want to stay close to me if he/she knew } \\
\text { the real me. }\end{array}$ & 21.3 & 21.3 & 23.4 & 10.6 & 23.4 \\
\hline I feel like I am unacceptable in basic ways. & 36.2 & 12.8 & 21.3 & 21.3 & 8.5 \\
\hline I believe that no one likes me. & 40.4 & 14.9 & 19.1 & 17 & 8.5 \\
\hline I feel like I am a failure as a person. & 29.8 & 12.8 & 17 & 14.9 & 25.5 \\
\hline I often feel that I am stupid. & 40.4 & 19.1 & 21.3 & 8.5 & 10.6 \\
\hline I think that I am useless. & 42.6 & 4.3 & 25.5 & 8.5 & 19.1 \\
\hline I think that I am able to do things as well as most other people. & 19.1 & 25.5 & 31.9 & 14.9 & 8.5 \\
\hline I think that I am able to succeed. & 21.3 & 25.5 & 25.5 & 12.8 & 14.9 \\
\hline I feel like I can't do anything right. & 27.7 & 17 & 23.4 & 23.4 & 8.5 \\
\hline I feel like I can't finish anything I am supposed to do. & 17 & 21.3 & 31.9 & 19.1 & 10.6 \\
\hline I feel like I am hopeless. & 38.3 & 10.6 & 17 & 19.1 & 14.9 \\
\hline I think I am incompetent when it comes to achievement. & 46.8 & 10.6 & 23.4 & 8.5 & 10.6 \\
\hline
\end{tabular}

item-subscale total correlations greater than 0.85 were removed due to content redundancy [40]. No items demonstrated item-total or item-subscale total correlations less than 0.30 [41]. Eight items demonstrated item-total or itemsubscale total correlations greater than 0.85 (Items $R, J, L, P$, $F F, M, Y, \& D D)$. Items were also checked for within-subscale inter-item correlations greater than 0.85 to reduce potential redundant items. Two item pairs were correlated above 0.85 . Items with high item-total, item-subscale, or inter-item correlations were examined for clarity, theoretical relevance to the construct of self-view, and change in each subscale's Cronbach's alpha if the item were removed (see Table 4). The full 33-item scale demonstrated excellent internal consistency in the clinical sample $(\alpha=0.98)$.

An item was considered for retention despite high item-total, item-subscale total, and inter-item correlations if a subscale's Cronbach's alpha value was greatly impacted by the removal of the item. Within the Self-Worth subscale, items $\mathrm{J}$ and FF demonstrated both item- total scale and item-subscale total correlations over 0.85 and were removed. Remov- 
Citation: Lehmann JK, Overholser JC, Athey A, et al. (2021) View of Self Scale: Measuring Negative Self-Referential Thoughts in Depression. Ann Cogn Sci 5(1):185-195

Table 4: Cronbach's alpha for each subscale overall and by dropping individual items.

\begin{tabular}{|c|c|c|}
\hline Subscale & Item & Cronbach's Alpha \\
\hline \multirow[t]{12}{*}{ Self-worth } & All & 0.95 \\
\hline & I feel like something is fundamentally wrong with me. & 0.95 \\
\hline & I think I am not important in any way. & 0.93 \\
\hline & I see myself as physically attractive. & 0.95 \\
\hline & I feel that I am worthless. & 0.93 \\
\hline & I think I am no good at all. & 0.94 \\
\hline & I have little respect for myself. & 0.94 \\
\hline & I like myself as a person. & 0.94 \\
\hline & I feel that I am a loser in every way. & 0.94 \\
\hline & I feel like I am flawed and defective. & 0.94 \\
\hline & I feel like I am unacceptable in basic ways. & 0.94 \\
\hline & I feel like I am hopeless. & 0.94 \\
\hline \multirow[t]{12}{*}{ Competence } & All & 0.94 \\
\hline & $\begin{array}{l}\text { I think that almost nothing I do at work (or school or household chores) is as good as what } \\
\text { other people can do. }\end{array}$ & 0.94 \\
\hline & I feel like I screw up everything I try. & 0.94 \\
\hline & I think that I do not have much to be proud of. & 0.94 \\
\hline & I feel like I am a failure as a person. & 0.93 \\
\hline & I often feel that I am stupid. & 0.94 \\
\hline & I think that I am useless. & 0.93 \\
\hline & I think that I am able to do things as well as most other people. & 0.95 \\
\hline & I think that I am able to succeed. & 0.94 \\
\hline & I feel like I can't do anything right. & 0.93 \\
\hline & I feel like I can't finish anything I am supposed to do. & 0.94 \\
\hline & I think I am incompetent when it comes to achievement. & 0.93 \\
\hline \multirow[t]{12}{*}{ Social Desirability } & All & 0.95 \\
\hline & I think I am a failure in my important relationships. & 0.94 \\
\hline & I feel that I deserve to be loved. & 0.95 \\
\hline & I feel that I am loved by friends, family, and/or my significant other. & 0.95 \\
\hline & I feel that I have let people down in some important ways. & 0.94 \\
\hline & I think I am too unlovable to reveal myself to other people. & 0.94 \\
\hline & When people show interest in me, I feel I am fooling them. & 0.94 \\
\hline & I think that I drive people away from me. & 0.94 \\
\hline & I believe that I am not worth of people's attention or affection. & 0.94 \\
\hline & I feel that I'm not lovable. & 0.94 \\
\hline & I believe that no one I desire would want to stay close to me if he/she knew the real me. & 0.94 \\
\hline & I believe that no one likes me & 0.94 \\
\hline
\end{tabular}

al of Item FF resolved one of the pairs of items with high inter-item correlations. The remaining pair of highly correlated items, $\mathrm{M}$ and $\mathrm{P}$, was examined further. Item $\mathrm{P}$ demonstrated an item-subscale total correlation over 0.85 whereas item $M$ did not demonstrate any high item-total, item-subscale total, or inter-item correlations. Item $\mathrm{P}$ was removed from the scale and item M was retained. Within the Social Desirability subscale, item $\mathrm{R}$ demonstrated both high item-total and item-subscale total correlations and was removed. Within the Competence subscale, item $Y$ demonstrated both high item-total and item-subscale total correlations and was removed. After removing items $\mathrm{R}, \mathrm{Y}, \mathrm{J}, \mathrm{FF}$, and $\mathrm{P}$, item-total and item-subscale total correlations were run again. No additional items were identified as having low item-total correlations or high item-total, item-subscale total, or inter-item correlations. The final measure consisted of 27 items.

To ensure the total scale was reduced appropriately, a 
Citation: Lehmann JK, Overholser JC, Athey A, et al. (2021) View of Self Scale: Measuring Negative Self-Referential Thoughts in Depression. Ann Cogn Sci 5(1):185-195

Table 5: Correlations examining relationships among view of self, depression severity, and life stress in depressed patients ( $n=47$ ) (below diagonal) and community adults $(n=39)$ (above diagonal).

\begin{tabular}{|l|l|l|l|l|l|}
\hline & VOSS & RSES & CTI-Self & PHQ-9 & MLES \\
\hline VOSS & & $-0.55^{* *}$ & $0.67^{* *}$ & $0.57^{* *}$ & 0.19 \\
\hline RSES & & $-0.71^{* *}$ & $-0.43^{* *}$ & -0.02 \\
\hline CTI-Self & $-0.91^{* *}$ & $-0.92^{* *}$ & & $0.42^{* *}$ & $0.32^{*}$ \\
\hline PHQ-9 & $0.91^{* *}$ & $-0.65^{* *}$ & $0.69^{* *}$ & & $0.45^{* *}$ \\
\hline MLES & $0.64^{* *}$ & -0.17 & 0.14 & 0.16 & \\
\hline
\end{tabular}

Note: ${ }^{* *} p<0.01$ and ${ }^{*} p<0.05$

correlation was run between the total 33-item scale and the reduced 27 -item scale. Correlation coefficients over 0.90 were deemed to represent a successful reduction of the overall measure. The total 33-item scale was highly correlated with the reduced 27 -item scale $(r=0.99, p<0.001)$. Similarly, the three total subscales were checked to see if they were reduced appropriately by running correlations between each original 11-item subscale and each reduced scale respectively. The reduced Self-Worth $(r=0.99, p<0.001)$, Competence $(r=0.99, p<0.001)$, and Social Desirability $(r=0.99, p<0.001)$ subscales each correlated with their respective total subscales at the level of 0.90 or higher.

The reliability of the reduced version of the VOSS was established by examining the internal consistency of the measure in both the clinical and community adult samples. The reduced version of the VOSS demonstrated excellent internal consistency within the clinical sample of veterans (Total: $\alpha=$ 0.97, Self-Worth: $\alpha=0.92$, Competence: $\alpha=0.91$, Social Desirability: $\alpha=0.92$ ) and adequate internal consistency within the community sample of adults (Total: $\alpha=0.87$, Self-Worth: $\alpha=0.74$, Competence: $\alpha=0.75$, Social Desirability: $\alpha=0.78$ ).

Means, standard deviations, and inter-correlations between the VOSS and other measures of self-view were examined to assess convergent validity of the VOSS in both the clinical sample and community samples (see Table 5). Correlations were run separately in both the clinical and community adult samples. In the clinical sample, there were strong, statistically meaningful correlations between VOSS scores and RSES scores $(r=-0.91, p<0.01)$ and CTI-Self subscale scores $(r=0.91, p<0.01)$. The RSES was also strongly, significantly correlated with the CTI-Self subscale $(r=-0.92, p<0.01)$ in the clinical sample. In the sample of community adults, VOSS scores were also significantly correlated with the RSES ( $r=$ -0.56, $p<0.01)$ and CTI-Self subscale $(r=0.69, p<0.01)$. The community sample RSES scores were also significantly correlated with CTI-Self subscale scores $(r=-0.71, p<0.01)$.

Discriminant validity of the VOSS was established through inter-correlations between the VOSS and Modified Life Experiences Survey. In the clinical sample, VOSS scores demonstrated a small, non-significant correlation with MLES scores ( $r=$ $0.25, p=0.09$ ). Similarly, in the sample of community adults, VOSS scores also demonstrated a small, non-significant correlation with a measure of life stress $(r=0.18, p=0.28)$. Inter-correlations between established measures of self-view and the MLES were also examined. The RSES demonstrated a small non-significant correlation with life stress in both the clinical $(r=-0.17, p=0.25)$ and community $(r=-0.02, p=0.89)$ samples. The CTI-Self subscale demonstrated a small non-significant correlation in the clinical sample $(r=0.14, p=0.34)$ and a medium significant correlation in the community sample $(r=0.32, p<0.05)$.

A series of correlations were conducted between scores on the VOSS, established measures of self-view (RSES and CTI-Self), and demographic variables with established relationships to self-view in order to examine criterion validity of the VOSS. Prior to running correlation analyses, categorical variables were dummy coded into dichotomous variables. Age was significantly related to view of self $(r=-0.33, p<$ 0.05 ) in the clinical sample, but not in the community sample. Older age was associated with fewer negative self-referential thoughts in the clinical sample. The total scale score for the VOSS was not correlated with any demographic variables in the community sample. Gender, employment status, education, and history of suicide attempt(s) were not significantly correlated with VOSS scores in either sample.

\section{View of self and depression}

VOSS scores were highly correlated with depression severity as measured by the PHQ-9 in both the clinical $(r=$ $0.65, p<0.01)$ and community $(r=0.60, p<0.01)$ samples. Established measures of self-view were also highly correlated with depression severity scores (see Table 5). Partial correlation was used to explore the relationship between self-view (VOSS) and depression severity (PHQ-9), while controlling for age within the clinical sample. In the clinical sample, there was a strong, positive, partial correlation between self-view and depression, controlling for age $(r=0.62, p<0.001)$ with high levels of negative self-referential thoughts being associated with greater depression severity. An inspection of zero order correlation ( $r=0.64$ ) suggested that controlling for age had very little effect on the strength of the relationship between these two variables.

Means, standard deviations, and inter-correlations were conducted to explore the relationships among depression severity scores and risk factors for depression and suicide. In the clinical sample, depression severity scores through the PHQ-9 demonstrated large correlations with measures of self-view, but not with age $(r=-0.23, p=0.11)$, gender $(r=0.07, p=$ $0.64)$, life stress $(r=0.16, p=0.29)$, history of past suicide attempt(s) $(r=-0.15, p=0.32)$, or education $(r=-0.08, p=0.62)$. In the community sample, depression severity scores demonstrated medium correlations with MLES scores $(r=0.45, p<$ 
Citation: Lehmann JK, Overholser JC, Athey A, et al. (2021) View of Self Scale: Measuring Negative Self-Referential Thoughts in Depression. Ann Cogn Sci 5(1):185-195

$0.01)$, age $(r=-0.37, p<0.05)$, and gender $(r=-0.33, p<0.05)$ and large correlations with measures of view of self ranging from $(r=0.42$ to $r=0.60, p<0.01)$. In the community sample, PHQ-9 scores were not correlated with history of past suicide attempt(s) $(r=0.21, p=0.22)$ or education $(r=0.13, p=0.43)$.

\section{Exploratory analyses}

Within the community sample, depression severity was significantly correlated with self- view, age, gender, and life stress. An exploratory hierarchical regression analysis was conducted to assess the ability of self-view to predict depression severity in the community sample after controlling for age, gender, and life stress (Table 5). In step $1=$, age and gender were entered into the model in order to determine how much variance in depression severity was accounted for by demographic variables. Demographic variables accounted for $20 \%$ of the variance in the model $\left(R^{2} \Delta=0.20, F \Delta(2,36)=4.55\right.$, $p<0.05$ ). In step 2 , ratings of life stress (MLES) were added to assess the relationship between life stress and depression severity. Levels of life stress accounted for an additional $12 \%$ of the variance in depression severity $\left(R^{2} \Delta=0.12, F \Delta(1,35)=\right.$ $6.45, p<0.01)$. In step 3 , VOSS scores were entered to assess the relationship between self-view and depression severity. Self-view accounted for an additional $22 \%$ of the variance in depression severity $\left(R^{2} \Delta=0.22, F \Delta(1,34)=16.81, p<0.001\right)$.

\section{Discussion}

The View of Self Scale is a brief measure of negative self-referential thoughts, an important component in the clinical experience of depression [42]. In the current study, the VOSS demonstrated strong psychometric properties in a sample of psychiatric outpatients with depression from a Veterans Affairs Medical Center $(n=47)$ and a sample of community adults utilizing food pantries in a large Midwestern city $(n=39)$. Clinicians can use the VOSS for initial assessment and treatment planning with depressed patients. The VOSS extends beyond other measures of self-view by providing a theory-driven measure with more thorough assessment of clinically relevant theoretical domains associated with the construct of self-view. The VOSS improves on current measures of self-view by providing a measure that is easy to administer and has a clear and simple scoring system.

The VOSS displayed strong psychometric properties in a sample of depressed patients and a sample of community adults. Initial evidence of internal consistency, convergent validity, discriminant validity, and criterion validity were established by the present study. The VOSS demonstrated excellent internal consistency in the clinical sample and adequate internal consistency in the non-clinical sample. The VOSS was highly correlated with measures of self-esteem and view of self and performed similarly to the RSES and CTI-Self subscale when compared to a number of different demographic predictor variables and when compared across both samples. As expected, participants in the clinical sample had a lower self-view across all three measures when compared with the community adult group.

Convergent validity of the VOSS was established by comparing View of Self scores to established measures of self-view. While the VOSS was highly correlated to both the Rosenberg Self-Esteem Scale and the CTI-Self subscale across both samples, the strength of correlation among the measures was notably lower in the community sample. It is possible that lower correlations among measures of self-view in the community sample reflect that view of self is a more salient construct within a clinical sample than within a community adult sample.

Specifically, the observed group differences could further emphasize the strong relationship between depression severity and negative self-referential thoughts [43].

Discriminant validity of the VOSS was established by comparing View of Self scores to life stressors. As the VOSS aims to measure trait self-esteem rather than state self-esteem, scores were not expected to be related to the presence or absence of life stressors. As expected, the VOSS demonstrated a non-significant relationship with life stress in both samples. In contrast, the CTI-Self subscale demonstrated a significant medium correlation with measures of life stress in the community adult sample. It may be that the CTI-Self subscale includes items which reflect more of a state-level measure of self-esteem, which is susceptible to fluctuations in life stress or mood, rather than a trait-level measure of self-esteem [44].

In the present study, self-view exhibited large correlations with depression severity in both depressed patients and community adults. This finding supports the claim that negative self-view is an important feature of depression [10]. Individuals with low self-esteem are more likely to develop depression [45]. Additionally, many individuals with depression also experience a lasting drop in self-esteem after experiencing an initial depressive episode [46]. Multiple theories of depression include self-view as a key factor contributing to the development of depression $[47,48]$. Explicit self-esteem ratings improve with the reduction of depression symptoms following integrative treatment for depression including a combination of psychotherapy and psychotropic medication [49]. This well-established relationship further emphasizes the importance of developing self-view assessment tools both with and for individuals with depression. Similarly, these results highlight the importance of utilizing interventions focused on self-view when working with depressed patients.

Self-view, as measured by the VOSS, was significantly related to age in the clinical sample, but not in the community sample. In the clinical sample, older age was associated with fewer negative self-referential thoughts. In the community adult sample, depression severity scores were significantly related to self-view, age, sex, and life stressors. Higher depression severity scores were associated with female sex, more negative self-referential thoughts, younger age, and a greater amount of life stress. As expected based on the literature, older adults in the community sample exhibited fewer symptoms of depression than younger adults.

In the clinical sample, depression severity scores were significantly related to self-view and not significantly related to age or life stress. While stressful life events are implicated in vulnerability for depression, the vulnerability associated 
Citation: Lehmann JK, Overholser JC, Athey A, et al. (2021) View of Self Scale: Measuring Negative Self-Referential Thoughts in Depression. Ann Cogn Sci 5(1):185-195

with a single stressful event may only increase risk for a depressive episode within one month after the event [50]. The non-significant relationship between depression severity and age may also be related to the presence of a higher number of individuals with a chronic course of depression within the clinical sample.

Theoretically-derived self-assessment tools of self-view may be useful in establishing goals and assessing progress in psychotherapy for depression. Cognitive behavioral interventions have been effective in improving self-esteem and reducing symptomology across psychiatric diagnoses including depression [51]. Measures such as the VOSS can be useful in identifying negative automatic thoughts or cognitive distortions within the therapy context. Following assessment, cognitive behavioral strategies can be used to help the client identify distortions, challenge negative automatic thoughts, and create alternative thoughts which are more accurate and helpful. Intervention for improving self-esteem in individuals with depression can include expanding the client's perspective, increasing frequency of self-reinforcement, improving coping strategies, and revising the goals and standards the client uses to evaluate themselves [52].

Perfectionism associated with unrealistic goals and standards is associated with various forms of psychopathology including depression and can present an additional target for intervention within psychotherapy [53].

The results from the present study should be considered in light of a number of limitations. First, the cross-sectional design of the study limits the conclusions which can be drawn from the data. For example, it has been assumed that a negative view of self precedes the onset and influences the course of a depressive episode. However, it is equally plausible that a negative view of self is best seen as a cognitive and interpretive symptom of depression that subsides when the depression recedes. Additionally, as family mental health history was not assessed, the current study is unable to speak to genetic components of depression. Second, only the veteran population completed the SCID diagnostic interview. While the community adult sample was screened for depression and substance use disorders, participants were not screened for other psychological or medical disorders. Third, the clinical sample was largely composed of males, which limits the generalizability of the results. Finally, the current study was unable to analyze longitudinal data to see if the VOSS is sensitive to change over time and related mental health outcomes. View of self plays an important role in the etiology, experience of, and recovery from depression. Negative self-referential thoughts provide an important target for clinical interventions in the treatment of patients with depression. The VOSS provides a new, theory- based measure of self-view, and in the present study, demonstrated strong psychometric properties in patients diagnosed with depression and community adults. Measures such as the VOSS can be used to identify negative self-views held by patients diagnosed with depression or identify individuals at greater risk for developing depression.

\section{References}

1. Cheng H, Furnham A (2004) Perceived parental rearing style, self-esteem and self-criticism as predictors of happiness. Journal of Happiness Studies 5: 1-21.

2. Baumeister RF, Campbell JD, Krueger JI, et al. (2003) Does high self-esteem cause better performance, interpersonal success, happiness, or healthier lifestyles? Psychol Sci Public Interest 4: 1-44.

3. O'Donnell K, Brydon L, Wright CE, et al. (2008) Self-esteem levels and cardiovascular and inflammatory responses to acute stress. Brain, Behavior, and Immunity 22: 1241-1247.

4. Forthofer MS, Janz NK, Dodge JA, et al. (2001) Gender differences in the associations of self-esteem, stress and social support with functional health status among older adults with heart disease. J Women Aging 13: 19-37.

5. Crocker J, Park LE (2004) The costly pursuit of self-esteem. Psychol Bull 130: 392-414.

6. Moritz S, Veckenstedt R, Randjbar S, et al. (2010) Course and determinants of self-esteem in people diagnosed with schizophrenia during psychiatric treatment. Psychosis 2: 144-153.

7. Vater A, Ritter K, Schröder AM, et al. (2013) When grandiosity and vulnerability collide: Implicit and explicit self-esteem in patients with narcissistic personality disorder. J Behav Ther Exp Psychiatry 44: 37-47.

8. Beck AT, Brown G, Steer R, et al. (2001) Psychometric properties of the beck self-esteem scales. Behaviour Research and Therapy 39: 115-124.

9. Beck JS (1995) Cognitive therapy: Basics and beyond. Guilford Press, New York.

10. Sowislo J, Orth U (2013) Does low self-esteem predict depression and anxiety? A meta-analysis of longitudinal studies. Psychol Bull 139: 213-240.

11. Klein D, Kotov R, Bufferd S (2011) Personality and depression: Explanatory models and review of the evidence. Annu Rev Clin Psychol 7: 269-295.

12. Franck E, De Raedt R (2007) Self-esteem reconsidered: Unstable self-esteem outperforms level of self-esteem as vulnerability marker for depression. Behaviour Research and Therapy 45: 1531-1541.

13. Bhar S, Ghahramanlou HM, Brown G, et al. (2008) Self-esteem and suicide ideation in psychiatric outpatients. Suicide Life Threat Behav 38: 511-516.

14. Jeon H, Jon DI, Hong HJ, et al. (2013) The relationship between low self-esteem and suicide attempt in patients with major depressive disorder: A pilot study. Korean Journal of Psychopharmacology 24: 180-185.

15. Overholser JC, Adams D, Lehnert K, et al. (1995) Self-esteem deficits and suicidal tendencies among adolescents. J Am Acad Child Adolesc Psychiatry 34: 919-928.

16. First MB, Spitzer RL, Gibbon M, et al. (1995) Structured clinical interview for DSM-IV-TR axis I disorders, research version, patient edition. (SCID-I/P): Biometrics Research, New York State Psychiatric Institute, New York.

17. American Psychiatric Association (1994) Diagnostic and statistical manual of mental disorders. ( $4^{\text {th }}$ edn), American Psychiatric Publishing, VA, Arlington.

18. Kessler R, Chiu W, Demler O, et al. (2005) Prevalence, severity, and comorbidity of 12-month DSM-IV disorders in the national comorbidity survey replication. Arch Gen Psychiatry 62: 617-627. 
Citation: Lehmann JK, Overholser JC, Athey A, et al. (2021) View of Self Scale: Measuring Negative Self-Referential Thoughts in Depression. Ann Cogn Sci 5(1):185-195

19. Schneider B, Maurer K, Sargk D, et al. (2004) Concordance of DSM-IV axis I and II diagnoses by personal and informant's interview. Psychiatry Res 127: 121-136.

20. Lobbestael J, Leurgans M, Arntz A (2011) Inter-rater reliability of the structured clinical interview for DSM-IV axis I disorders (SCID I) and axis II disorders (SCID II). Clin Psychol Psychother 18: 75-79.

21. Keith LK, Bracken BA (1996) Self-concept instrumentation: A historical and evaluative review. Handbook of self-concept: Developmental, social, and clinical considerations, Wiley, New York.

22. Young J, Behary WT (1998) Schema-focused therapy for personality disorders. In: N Tarrier, A Wells, G Haddock, Treating complex cases: The cognitive behavioural therapy approach. (edn), Wiley series in clinical psychology. John Wiley, New York, 340-376.

23. Beckham EE, Leber WR, Watkins JT, et al. (1986) Development of an instrument to measure Beck's cognitive triad: The cognitive triad inventory. J Consult Clin Psychol 54: 566-567.

24. Rosenberg $M$ (1965) Society and the adolescent self-image. Princeton University Press, Princeton, NJ.

25. Beck AT, Brown G, Steer RA, et al. (1987) Differentiating anxiety and depression: $A$ test of the cognitive content-specificity hypothesis. J Abnorm Psychol 96: 179-183.

26. Anderson KW, Skidmore JR (1995) Empirical analysis of factors in depressive cognition: The cognitive triad inventory. J Clin Psychol 51: 603-609.

27. Pössel P (2009) Cognitive triad inventory (CTI): Psychometric properties and factor structure of the German translation. J Behav Ther Exp Psychiatry 40: 240-247.

28. McIntosh CN, Fischer DG (2000) Beck's cognitive triad: One versus three factors. Canadian Journal of Behavioural Science 32 153-157.

29. Tinakon W, Nahathai W (2012) A comparison of reliability and construct validity between the original and revised versions of the rosenberg self-esteem scale. Psychiatry Investig 9: 54-58.

30. Hatcher J, Hall LA (2009) Psychometric properties of the Rosenberg self-esteem scale in African American single mothers. Issues Ment Health Nurs 30: 70-77.

31. Brown GW, Bifulco A, Veiel HO, et al. (1990) Self-esteem and depression. II. Social correlates of self-esteem. Soc Psychiatry Psychiatr Epidemiol 25: 225-234.

32. Andrews B, Brown GW (1993) Self-esteem and vulnerability to depression: The concurrent validity of interview and questionnaire measures. J Abnorm Psychol 102: 565-572.

33. Kroenke K, Spitzer RL, Williams JB (2001) The PHQ-9: Validity of a brief depression severity measure. J Gen Intern Med 16: 606613.

34. Manea L, Gilbody S, McMillan D (2012) Optimal cut-off score for diagnosing depression with the patient health questionnaire (PHQ-9): A meta-analysis. CMAJ 184: E191-E196.

35. Cameron IM, Crawford JR, Lawton K, et al. (2008) Psychometric comparison of PHQ-9 and HADS for measuring depression severity in primary care. Br J Gen Pract 58: 32-36.

36. Sarason IG, Johnson JH, Siegel JM (1978) Assessing the impact of life changes: Development of the life experiences survey. J Consult Clin Psychol 46: 932-946.

37. Little RJ (1988) A test of missing completely at random for multivariate data with missing values. Journal of the American Statistical Association 83: 1198-1202.

38. Kaiser HF (1974) An index of factorial simplicity. Psychometrika 39: 31-36.

39. Bartlett MS (1954) A note on the multiplying factors for various chi square approximations. Journal of the Royal Statistical Society 16: 296-298.

40. Netemeyer R, Burton S, Biswas D, et al. (2002) Psychometric properties of shortened versions of the automatic thoughts questionnaire. Educational and Psychological Measurement 62: 111-129.

41. Ferketich S (1991) Focus on psychometrics: Aspects of item analysis. Res Nurs Health 14: 165-168.

42. Beck AT, Rush AJ, Shaw BF, et al. (1979) Cognitive therapy of depression. Guilford Press, New York.

43. Ehret AM, Joormann J, Berking M (2015) Examining risk and resilience factors for depression: The role of self-criticism and self-compassion. Cogn Emot 29: 1496-1504.

44. Clasen PC, Fisher AJ, Beevers CG (2015) Mood-reactive self-esteem and depression vulnerability: Person-specific symptom dynamics via smart phone assessment. PLoS One 10: e0129774.

45. Steiger A, Fend H, Allemand M (2015) Testing the vulnerability and scar models of self- esteem and depressive symptoms from adolescence to middle adulthood and across generations. Dev Psychol 51: 236-247.

46. Shahar G, Davidson L (2003) Depressive symptoms erode self-esteem in severe mental illness: A three-wave, cross-lagged study. J Consult Clin Psychol 71: 890-900.

47. Abramson LY, Seligman ME, Teasdale JD (1978) Learned helplessness in humans: Critique and reformulation. J Abnorm Psychol 87: 49-74.

48. Beck AT (1967) Depression: Clinical experimental and theoretical aspects. Harper \& Row, New York.

49. Wegener I, Geiser F, Alfter S (2015) Changes of explicitly and implicitly measured self- esteem in the treatment of major depression: Evidence for implicit self-esteem compensation. Comprehensive Psychiatry 58: 57-67.

50. Kendler K, Gardner CO (2016) Depressive vulnerability, stressful life events and onset of major depression: A longitudinal model. Psychol Med 46: 1865-1874.

51. Kolunbinski DC, Frings D, Nikčević AV, et al. (2018) A systematic review and meta-analysis of $C B T$ interventions based on the fennell model of low self-esteem. Psychiatry Res 267: 296-305.

52. Overholser JC (1996) Cognitive-behavioral treatment of depression, part V: Enhancing self- esteem and self-control. Journal of Contemporary Psychotherapy 26: 163-176.

53. Limburg K, Watson HJ, Hagger MS, et al. (2017) The relationship between perfectionism and psychopathology: A meta-analysis. J Clin Psychol 73: 1301-1326.

DOI: $10.36959 / 447 / 351$ 